Special Issue of the 6th International Congress \& Exhibition (APMAS2016), Maslak, Istanbul, Turkey, June 1-3, 2016

\title{
Comparison of Quasi-Static Constitutive Equations and Modeling of Flow Curves for Austenitic 304 and Ferritic 430 Stainless Steels
}

\author{
B. SEnER* AND M.E. YurCI \\ Yildiz Technical University, Department of Mechanical Engineering, 34349 Istanbul, Turkey
}

\begin{abstract}
Four different quasi-static flow curve models were investigated to describe flow curves of austenitic (304) and ferritic (430) stainless steel sheets. Uni-axial tensile tests were carried out and material constants of the models were determined by curve fitting technique. Applicability of these models over the uniform plastic deformation region was evaluated according to the value of nonlinear regression parameter $R^{2}$. It was observed, that the predictions made by El-Magd model are in good agreement with the experimental data for both 304 and 430 stainless steel sheets.
\end{abstract}

DOI: 10.12693/APhysPolA.131.605

PACS/topics: $81.05 . \mathrm{Bx}$

\section{Introduction}

Constitutive equations give a mathematical representation of the flow stress behavior of materials and involve a particular number of material constants, which are evaluated using limited number of experimental data [1]. These equations are used in finite element (FE) modeling software to simulate the material response under specified loading conditions [2]. Therefore, the accuracy of the numerical simulation largely depends on how accurately the deformation behavior of the material is being represented by the constitutive equation [3]. Many researchers have conducted studies on modeling of flow curves of materials. Singh [4] has investigated suitability of the different constitutive equations for different grain size and temperatures in 316L austenitic stainless steel. Lemoine et al. [5] have compared different constitutive equations for DP 780 and TRIP 780 steel sheets and have recommended the most suitable model for these materials. Li et al. [6] have researched the constitutive relationship of boron steel for the numerical simulation of hot stamping and have attained equations based on strain, strain rate and temperature. In the present work, a comparative study was carried out on Hollomon, Ludwik, Swift and El-Magd models to represent the flow behavior of austenitic (304) and ferritic (430) stainless steel sheets. Uni-axial tensile tests were carried out to determine the material constants of models. Subsequently, the suitability of these models was evaluated by comparing nonlinear regression parameter $R^{2}$ and the most suitable model was determined for the test materials.

\section{Constitutive laws}

Constitutive equation is used as a material parameter input to the FE software. Normally, constitutive

\footnotetext{
*corresponding author; e-mail: borasen@yildiz.edu.tr
}

equation is a mathematical representation of relationship between the flow behavior of material and process parameters like plastic strain $\varepsilon_{\mathrm{p}}$, strain rate $\dot{\varepsilon}$ and temperature $T$ [7]. Constitutive equations can be broadly categorized into two groups: quasi-static and dynamic flow curve models. In quasi-static models the flow stress is a function of plastic strain, whereas in dynamic models it is a function of plastic strain, strain rate and temperature. In this work, quasi-static flow curve models were investigated to predict strain hardening characteristics of the austenitic (304) and ferritic (430) stainless steels. Dynamic flow curve models were not investigated in this study because both of the materials have low strain rate sensitivity and forming process is carried out at room temperature.

Table I shows quasi-static models which are investigated in this study.

TABLE I

Equations of considered quasi-static models.

\begin{tabular}{c|cc}
\hline \hline Model & Equation \\
\hline Hollomon & $\sigma(\varepsilon)=K \varepsilon_{\mathrm{p}}^{n}$ & $(1)$ \\
Ludwik & $\sigma(\varepsilon)=\sigma_{\mathrm{y}}+K \varepsilon_{\mathrm{p}}^{n}$ & $(2)$ \\
Swift & $\sigma(\varepsilon)=K\left(\varepsilon_{\mathrm{p}}+\varepsilon_{0}\right)^{n}$ & $(3)$ \\
El-Magd & $\sigma(\varepsilon)=A+B \varepsilon_{\mathrm{p}}+C\left[1-\exp \left(-\beta \varepsilon_{\mathrm{p}}\right)\right]$
\end{tabular}

Here $K$ is the strength coefficient, $\varepsilon_{\mathrm{p}}$ plastic strain, $\sigma_{y}$ is the yield stress, $n$ is the strain hardening exponent, $\varepsilon_{0}$ is initial strain, $A, B, C$ and $\beta$ are the material constants.

\section{Experimental work}

Tensile tests were carried out for determination of flow curves of 304 and 430 stainless steel sheets. The specimens were prepared according to ASTM E8M standard specification, by laser cutting. The thicknesses of the 304 and 430 stainless steel sheets were 0.8 and $0.7 \mathrm{~mm}$, respectively. $50 \mathrm{kN}$ load capacity universal tensile testing machine was used. The experiments were conducted at 
constant strain rate $\left(\dot{\varepsilon}=0.001 \mathrm{~s}^{-1}\right)$ in all directions. A computer control system was used to record the force vs. extension data, where extension was measured using a video-type extensometer in the experiments. Primarily, engineering stress vs. engineering strain curve was obtained by converting the force-extension curve. Subsequently true stress-true strain curve was acquired and finally true stress-plastic strain curve was obtained by removing the elastic region from the true stress-true strain curve.

Elasticity modulus, $E$, was calculated by dividing the tensile stress by the strain in the elastic portion (linear region) of the stress-strain curve:

$$
E=\frac{\sigma}{\varepsilon}=\frac{F / A_{0}}{\Delta L / L_{0}},
$$

where $F$ is the force exerted on a sample under tension, $A_{0}$ is the original cross-sectional area through which the force is applied, $\Delta L$ is the amount by which the length of the sample changes and $L_{0}$ is the original length of the object.

Elastic strain $\left(\varepsilon_{\mathrm{e}}\right)$ is given by:

$$
\varepsilon_{\mathrm{e}}=\frac{\sigma}{\varepsilon} \text {. }
$$

Then plastic strain $\left(\varepsilon_{\mathrm{p}}\right)$ can be calculated by subtraction of elastic strain from total true strain:

$$
\varepsilon_{\mathrm{p}}=\varepsilon_{\mathrm{t}}-\varepsilon_{\mathrm{e}} .
$$

The yield stress was obtained by means of an offset to $0.2 \%$ of strain, related to the straight part of the elastic stage.

\section{Results and discussion}

Four different constitutive models (Hollomon, Ludwik, Swift and El-Magd) are evaluated in this work. The parameters for such models are identified from experimentally determined flow curves of materials by curve fitting techniques. Nonlinear least square regression method and Levenberg-Marquardt algorithm were used. The same fitting strain range between yield and tensile strength has been chosen for all models. Comparisons of predicted flow curves by different quasi-static models with experimental data for 304 and 430 stainless steel sheets are shown in Figs. 1 and 2, respectively.

Values of material parameters found for each of the models for 304 and 430 stainless steels are shown in Table II and Table III, respectively.

TABLE II

Parameters obtained for the constitutive equations, during the characterization of the 304 stainless steel, in the rolling direction.

\begin{tabular}{c|c|c|c|c|c|c}
\hline \hline Models & $\begin{array}{c}\mathrm{K} \\
{[\mathrm{MPa}]}\end{array}$ & $\mathrm{n}$ & $\mathrm{A}$ & $\mathrm{B}$ & $\mathrm{C}$ & $\beta$ \\
\hline Hollomon & 1532 & 0.429 & - & - & - & - \\
Ludwik & 1126 & 0.6 & - & - & - & - \\
Swift & 1549 & 0.443 & - & - & - & - \\
El-Magd & - & - & 340.6 & 1757 & 60.3 & 24.36
\end{tabular}
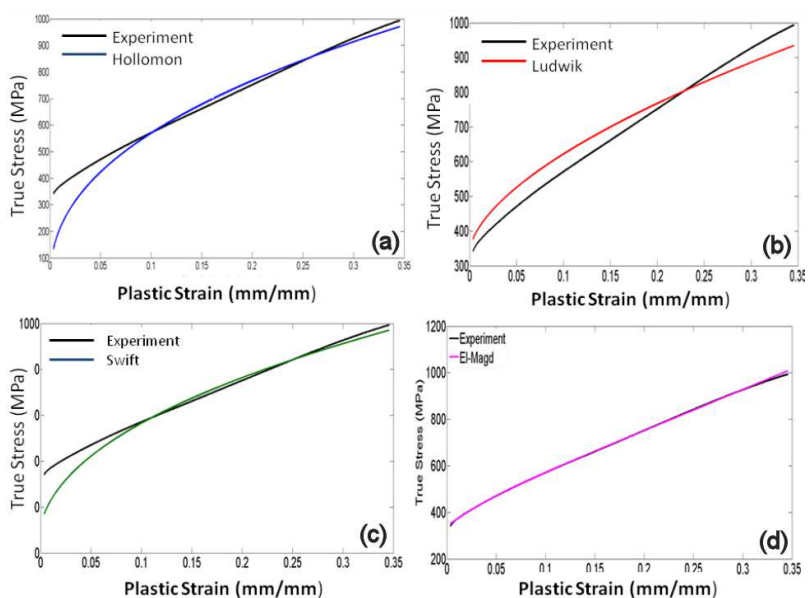

Fig. 1. Comparison of predicted flow curves by different quasi-static models with experimental data for austenitic 304 stainless steel.
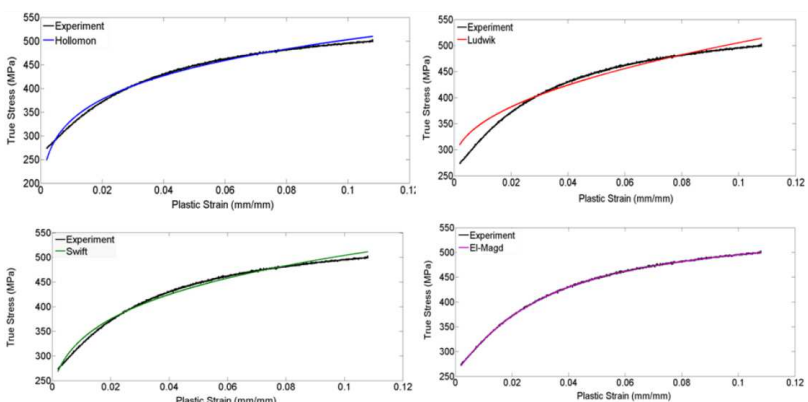

Fig. 2. Comparison of predicted flow curves by different quasi-static models with experimental data for ferritic 430 stainless steel.

Analysis of Tables II and III shows, that $n$ value of the 304 stainless steel is higher than that of 430 stainless steel sheet for each equation. When studying the true stress versus plastic strain, Figs. 1 and 2, it appears that the ferritic stainless steel has maximum strain in the plastic region around 0.11 , whereas the austenitic stainless steel around the value of 0.35 . This can be explained by observed $n$ value, because $n$ is an important factor in the distribution of strain, that is, it has great influence on the ductility of the material [8]. The predictability of the

TABLE III

Parameters obtained for the constitutive equations, during the characterization of the 430 stainless steel, in the rolling direction.

\begin{tabular}{c|c|c|c|c|c|c}
\hline \hline Models & $\begin{array}{c}\mathrm{K} \\
{[\mathrm{MPa}]}\end{array}$ & $\mathrm{n}$ & $\mathrm{A}$ & $\mathrm{B}$ & $\mathrm{C}$ & $\beta$ \\
\hline Hollomon & 759.5 & 0.179 & - & - & - & - \\
Ludwik & 685 & 0.469 & - & - & - & - \\
Swift & 783.2 & 0.193 & - & - & - & - \\
El-Magd & - & - & 256.1 & 425.9 & 201.2 & 37.84
\end{tabular}


constitutive equations is evaluated by employing nonlinear regression $R^{2}$ parameter. This statistical parameter measures how successful the fit is in explaining the variation of the data. $R$-square is the square of the correlation between the response values and the predicted response values [9].

$R^{2}$ values of the models for 304 and 430 stainless steel sheets are given in Fig. 3 and 4 respectively. It can be seen that El-Magd is the best model in the prediction of flow curve for both 304 and 430 stainless steels. When compared with the other models, El-Magd model has exponential term, while the other models (Hollomon, Ludwik and Swift) have a single set of parameters. This term increases ability of fitting the experimental stressstrain curves of the materials. Therefore, El-Magd model had the highest $R^{2}$ values among the other quasi-static models.

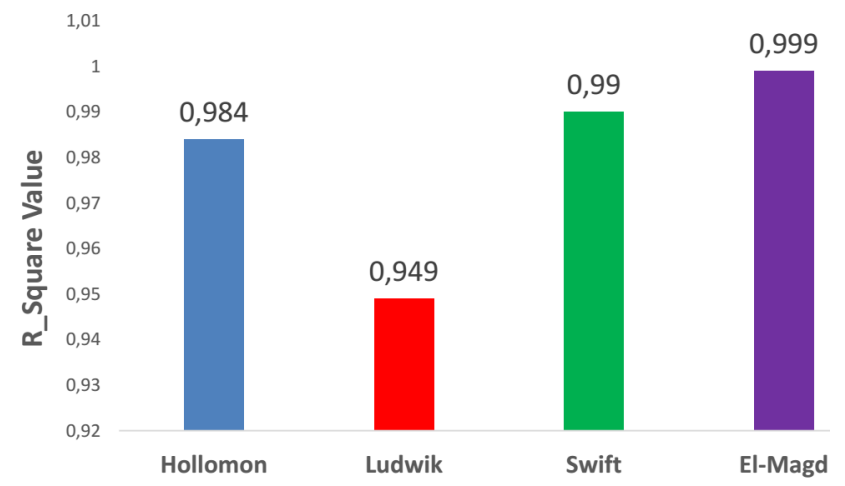

Fig. 3. $\quad R^{2}$ values of the different constitutive models for 304 stainless steel sheet.

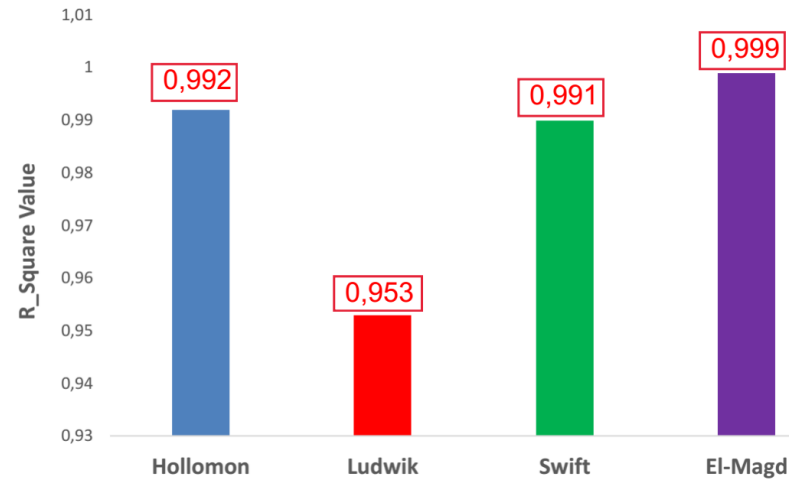

Fig. 4. $R^{2}$ values of the different constitutive models for 430 stainless steel sheet.

\section{Conclusions}

Based on the analysis done and on experimental results, the following conclusions can be made:
1. Maximum plastic strain value of the austenitic 304 stainless steel sheet (0.35) is higher than that of the 430 ferritic stainless steel sheet $(0.11)$ in the uniform plastic deformation region, due to the higher $n$ value.

2. From nonlinear regression parameter $R^{2}$ values, it can be concluded that for predicting the flow stress of 304 and 430 sheet materials in the uniform plastic deformation region, El-Magd model is highly effective, compared to the other three models, namely Hollomon, Ludwik and Swift. Therefore, usage of this model in FEM software is important for accuracy of the sheet metal forming simulations.

3. Swift model is more successful than the other two models (Hollomon and Ludwik) for austenitic (304) stainless steel, whereas Hollomon and Swift models gave similar results for ferritic (430) stainless steel.

4. Ludwik model has shown the worst performance for both of the materials.

\section{Acknowledgments}

This research has been supported by Yildiz Technical University Scientific Research Projects Coordination Department. Project Number: 2014-06-01-DOP03.

\section{References}

[1] R. Liang, A.S. Khan, Int. J. Plasticity 15, 963 (1999).

[2] X. He, Z. Yu, X. Lai, Comp. Mater. Sci. 44, 760 (2008).

[3] Y.C. Lin, X-M. Chen, Mater. Des. 32, 1733 (2011).

[4] K.K. Singh, Mater. Sci. Tech.-Lond. 20, 1134 (2004).

[5] X. Lemoine, S. Sriram, R. Kergen, AIP Conf. Proc. 1353, 1417 (2011).

[6] H. Li, L. He, G. Zhao, L. Zhang, Mater. Sci. Eng. A 580, 330 (2013).

[7] S.K. Paul, Comp. Mater. Sci. 65, 91 (2012).

[8] A.K. Ghosh, J. Eng. Mater. Technol. 99, 264 (1977).

[9] D. G. Kleinbaum, L.L. Kupper, Applied Regression Analysis and Other Multivariable Methods, Cengage Learning, Belmont 2007. 